\title{
IMPORTANCIA DE LA SIMULACIÓN CLÍNICA EN EL DESARROLLO PERSONAL Y DESEMPEÑO DEL ESTUDIANTE DE ENFERMERÍA ${ }^{1}$
}

\author{
IMPORTANCE OF CLINICAL SIMULATION IN THE PERSONAL \\ DEVELOPMENT AND PERFORMANCE OF THE NURSING STUDENT
}

\section{IMPORTÂNCIA DA SIMULAÇÃO CLÍNICA NO DESENVOLVIMENTO PESSOAL E DESEMPENHO DO ESTUDANTE DE ENFERMAGEM}

\author{
Vinka Aleova Yusef Contreras* \\ Gustavo Adolfo Sanhueza Ríos** \\ Fredy Andrés Seguel Palma***
}

RESUMEN

Objetivos: Determinar la importancia de la Simulación Clínica sobre el desempeño del estudiante de enfermería en campo clínico e indagar los efectos que produce la metodología para su posterior desempeńo en contextos reales. Material y Método: Estudio mixto de abordaje cuantitativo transversal, de asociación y cualitativo fenomenológico. La unidad de análisis fueron estudiantes de enfermería, la población correspondió al universo de dos cohortes de cuarto año de la Universidad Austral de Chile, 106 participantes para la aproximación cuantitativa y 10 para la cualitativa, seleccionados por muestreo de conveniencia. Los criterios de inclusión en ambas aproximaciones fueron haber cursado el $7^{\circ}$ y $8^{\circ}$ semestre de la carrera y participado en actividades de simulación de las asignaturas. La recolección de datos fue mediante cuestionarios sociodemográfico, de percepción tipo Likert (sometido a revisión de fachada y de claridad) y entrevistas en profundidad. Se efectuaron análisis descriptivos, pruebas paramétricas o no paramétricas mediante software SPSS v.15 y análisis cualitativo con reducción fenomenológica. Para cautelar la calidad de los datos se realizó triangulación por investigadores. El rigor ético fue resguardado con los principios de Ezequiel Emanuel, consentimiento informado y autorización del Comité Ético Científico de la Universidad. Resultados: La metodología fue valorada en ámbitos académicos y personales, aumentando la seguridad, habilidades, destrezas y presencia de memoria emocional en su desarrollo, favoreciendo el aprendizaje significativo. Conclusiones: Es importante la inserción temprana curricular de la simulación clínica, para potenciar y optimizar aspectos relacionados con los usuarios del sistema al interactuar en escenarios reales.

\footnotetext{
${ }^{1}$ Financiamimiento: Proyecto S-2015-77, Dirección de Investigación y Desarrollo, Universidad Austral de Chile.

*Enfermera, Mg en Enfermería, Instituto de Enfermería, Facultad de Medicina, Universidad Austral de Chile, Valdivia, Chile. ORCID: http://orcid.org/0000-0003-4972-9671 Email: vinkayusef@uach.cl Autora de correspondencia.

**Enfermero, Mg en Innovación de la Docencia Universitaria en Ciencias de la Salud, Instituto de Enfermería, Facultad de Medicina, Universidad Austral de Chile, Valdivia, Chile. ORCID: https://orcid.org/0000-0001-9400-8430 Email: gustavosanhueza@ uach.cl

***Enfermero, Doctor en Enfermería, Instituto de Enfermería, Facultad de Medicina, Universidad Austral de Chile, Valdivia, Chile. ORCID: http://orcid.org/0000-0001-7115-3651 Email: fredyseguel@uach.cl Autor de correspondencia.
} 
Palabras clave: Simulación de paciente; Estudiante de Enfermería; Competencia clínica; Entrenamiento simulado; Desarrollo personal; Desempeño.

\section{ABSTRACT}

Objective: To determine the importance of Clinical Simulation on the performance of the nursing student in the clinical field and to explore the effects of this methodology for their subsequent performance in real contexts. Material and Method: Mixed study, cross-sectional, quantitative, associative and qualitative phenomenological approach. The unit of analysis were nursing students; the population corresponded to the universe of two fourth-year cohorts from the Universidad Austral de Chile, 106 participants for the quantitative approach and 10 for the qualitative one, selected by convenience sampling. The inclusion criteria for both approaches were having attended the 7th and 8th semester of the study program and having participated in subject simulation activities. Data collection was carried out using sociodemographic, Likerttype perception questionnaires (after face validity and clarity review) and in-depth interviews. Descriptive analyzes, parametric or non-parametric tests were carried out using SPSS v.15 software and qualitative analysis with phenomenological reduction. Researcher triangulation was performed to ensure data quality. Ethical rigor was based on the principles of Ezequiel Emanuel, informed consent and authorization of the university's Scientific Ethics Committee. Results: The methodology was valued in academic and personal areas, increasing security, abilities, skills and the presence of emotional memory, thus contributing to meaningful learning. Conclusions: Early curricular implementation of clinical simulation is important to enhance and optimize aspects related to the users of the system when interacting in real scenarios.

Key words: Patient Simulation; Nursing Student; Clinical Competence; Simulated Training; Personal Development; Performance.

\section{RESUMO}

Objetivos: Determinar a importância da Simulação Clínica no desempenho do estudante de enfermagem no campo clínico e investigar os efeitos que a metodologia produz para seu posterior desempenho em contextos reais. Material e Método: Estudo misto, transversal, quantitativo, associativo e com abordagem fenomenológica qualitativa. A unidade de análise foi os estudantes de enfermagem, a população correspondeu ao universo de duas coortes do quarto ano da Universidade Austral do Chile, 106 participantes para a abordagem quantitativa e 10 para a qualitativa, selecionados por amostragem por conveniência. Os critérios de inclusão em ambas abordagens foram ter cursado o $7^{\circ}$ e $8^{\circ}$ semestres do curso e ter participado das atividades de simulação das disciplinas. A coleta de dados se deu por meio de questionários sociodemográficos, de percepção tipo Likert (submetidos a uma revisão de validade de face e clareza) e entrevistas em profundidade. Foram realizadas análises descritivas, testes paramétricos ou não paramétricos no software SPSS v.15 e análises qualitativas com redução fenomenológica. Para garantir a qualidade dos dados, a triangulação foi realizada por pesquisadores. O rigor ético foi resguardado pelos princípios de Ezequiel Emanuel, consentimento informado e autorização do Comitê de Ética Científica da universidade. Resultados: A metodologia foi valorizada no ambiente acadêmico e pessoal, aumentando a segurança, as habilidades e a presença da memória emocional no desenvolvimento pessoal, favorecendo a aprendizagem significativa. Conclusôes: A inserção curricular precoce da simulação clínica é importante para aprimorar e otimizar aspectos relacionados aos usuários do sistema na interação em cenários reais.

Palavras-chave: Simulação de paciente; Estudante de enfermagem; Competência clínica; Treinamento simulado; Desenvolvimento pessoal; Desempenho. 


\section{INTRODUCCIÓN}

El mundo universitario demanda la reorganización de enseñanzas en función del aprendizaje centrado en el estudiante, educación basada en competencias, introducción de nuevas metodologías activas y modelos que evalúen conocimientos, destrezas y habilidades, con el propósito de lograr en forma eficaz y duradera los objetivos formativos y competencias disciplinares ${ }^{(1)}$.

Referirse a campos de acción profesional implica comprender el concepto de competencia, referido a las habilidades y destrezas que demuestran tácitamente lo que un individuo es capaz de hacer en forma competente y responsable, concordante con el grado de preparación logrado, indispensables para la ejecución de labores propias de una determinada profesión $^{(2)}$.

A partir de este concepto se logran perfiles que definen el rango de acción y escenario de desempeño profesional. Sobre ello el Proyecto Tuning definió, desde el paradigma educativo, los diversos tipos de competencias disciplinares, relativas al saber, ser y hacer, divididas en dos perfiles: competencias genéricas, definidas como generales, transferibles y transversales a la trayectoria formativa, y competencias específicas que identifican el aprendizaje concreto $^{(3,4)}$.

Considerando lo anterior, es posible aseverar que en el área de la salud y en específico en enfermería, es necesario incluir metodologías que integren conocimientos en el contexto clínico, evaluando lo que el individuo sabe, sabe hacer y hace para evidenciar las competencias que se han adquirido a través del tiempo. Bajo esta premisa, la simulación clínica es necesaria en entornos de enseñanza aprendizaje, entrelazando conocimiento, habilidades y el factor humano ${ }^{(1)}$.

"Simular es representar algo, fingiendo o imitando lo que no es"(5); en el área de la salud consiste en posicionar al estudiante en un contexto que imite algún aspecto de la realidad, estableciendo situaciones similares a la práctica clínica ${ }^{(5)}$. Se encuadra con los lineamientos educacionales de muchas instituciones de enseñanza superior a nivel global, fomentando el constructivismo, enfoque centrado en el estudiante, con un rol principal en el desarrollo de competencias necesarias para el perfil de egreso ${ }^{(6,7)}$.

Tradicionalmente la enseñanza de habilidades era realizada directamente con el paciente, actualmente esta práctica es cuestionada, por la existencia de riesgos, a pesar de la supervisión docente, unido al derecho de los pacientes a recibir una intervención que considere la mejor opción disponible, incluyendo en este concepto la seguridad asistencial, teniendo en cuenta las consecuencias de los errores de la práctica sanitaria ${ }^{(8,9)}$.

En este contexto, es necesario destacar que el quehacer de la disciplina de enfermería se centra en la gestión de los cuidados de las personas, concepto que involucra aspectos éticos y legales relacionados con la seguridad del paciente, alineándose con las actuales políticas de calidad de atención a nivel nacional en los contextos de enseńanza aprendizaje, limitando el contacto estudiante-paciente ${ }^{(10,11)}$.

Por lo antes expuesto, surge la necesidad de incrementar el uso de la simulación clínica para potenciar, en el estudiante, aquellas destrezas y habilidades procedimentales y humanas, que le permitan mejorar su seguridad y capacidad de reacción ante situaciones desconocidas y de riesgo. Esto implica realizar entrenamiento en ambientes controlados, donde los errores forman parte del aprendizaje y se resuelven por medio de repeticiones, sin riesgo para la integridad del paciente. Se simulan situaciones o problemas que debe enfrentar, tal como ocurriría en un mismo escenario real, transformándose en una oportunidad favorable desde el punto de vista práctico, legal y ético ${ }^{(6,12)}$.

La expansión del uso de esta metodología obliga a realizar estudios para determinar el desarrollo e impacto de su utilización, con el fin de mantener y mejorar su calidad. En la enseñanza superior, es cada vez más frecuente observar a jóvenes desarrollándose en entornos de alta exigencia y tensión, que originan sentimientos negativos que dificultan su rendimiento y realización personal, provocando deterioro paulatino en su salud mental. El factor principal es el estrés que experimenta al enfrentarse al campo clínico, sin haber adquirido algún nivel de destreza que le dé seguridad para enfrentar la atención del paciente, disminuya el temor a equivocarse o a la posibilidad de que ocurra un error que, sin duda, afectan tanto al paciente, al propio estudiante y al docente a cargo ${ }^{(13)}$.

Por lo antes expuesto los objetivos de este estudio fueron: determinar la importancia de la Simulación Clínica sobre el desempeño del estudiante de enfermería en campo clínico e indagar los efectos 
que produce la metodología en el estudiante y en su posterior desempeño en contextos reales.

\section{MATERIAL Y MÉTODO}

Estudio mixto analítico, cuantitativo, transversal de asociación y cualitativo fenomenológico ${ }^{(14)}$, en el cual participaron estudiantes de enfermería de la Universidad Austral de Chile, no considerándose a estudiantes de otras carreras del área de la salud de la misma universidad, por las escasas o inexistentes actividades de simulación implementadas en sus currículos, al momento de la ejecución del estudio.

Para la aproximación cuantitativa la población estuvo conformada por un universo de 106 estudiantes, aun cuando el n para algunas variables fluctuó en base a la cantidad de respuestas realizadas por los participantes que pertenecían a las cohortes de cuarto ańo de la carrera de enfermería, en los años 2015 y 2016. Los criterios de inclusión fueron que hayan cursado el $7^{\circ}$ y $8^{\circ}$ semestre de la carrera y que hayan participado en actividades de simulación clínica incorporadas en los programas de las asignaturas de dichos niveles de formación, cautelando con ello que todos los participantes se encontraran en igualdad de condiciones y de oportunidades, con relación a la participación en a las experiencias de simulación.

Los datos fueron recolectados por medio de 2 instrumentos de elaboración propia: un "Cuestionario sociodemográfico" que midió 8 variables con respuestas categorizadas (Tabla 1) y un "Cuestionario de evaluación de percepción sobre utilización metodología simulación clínica”, escala tipo Likert ordinal, con aseveraciones, cuyas respuestas categóricas variaban desde totalmente en desacuerdo (1 punto), hasta totalmente de acuerdo (5 puntos), con un total de 75 puntos. Las 15 aseveraciones contenidas en el instrumento fueron las siguientes: 1) Después de empezar a utilizar esta metodología sentí que mi forma de enfrentar las situaciones en la práctica clínica había evolucionado positivamente; 2) La simulación me ha ayudado a integrar teoría y práctica; 3) Me sentí motivado con la metodología; 4) Me sentí comprometido con mi auto aprendizaje; 5) La experiencia con el simulador ha aumentado mi seguridad a la hora de enfrentarme al desempeño en el campo clínico; 6) La metodología utilizada ayuda a desarrollar el razonamiento crítico y la toma de decisiones; 7) La simulación ha aumentado la confianza en mis habilidades técnicas; 8) La metodología utilizada colaboró en el logro de mis objetivos de aprendizaje; 9) Las experiencias vividas con la aplicación de la metodología fueron importante en mi aprendizaje; 10) La interacción con la simulación ha mejorado mi competencia clínica, en relación al grado de desarrollo de habilidades y destrezas adquiridas; 11) Me sentí estimulado para desarrollar las tareas propuestas en el desarrollo de la metodología; 12) La utilización de la metodología ha contribuido a reconocer la responsabilidad social que me compete; 13) La utilización de la metodología contribuyo a mejorar mi autoestima en relación a mi desempeño frente a situaciones complejas en la práctica clínica; 14) En general, la experiencia de trabajar con la simulación clínica ha sido satisfactoria; 15) La utilización de esta metodología debería incluirse en las mallas curriculares de las carreras del área de la salud en forma permanente.

Este cuestionario fue sometido a revisión de fachada ${ }^{(15)}$ por 3 docentes expertos con formación en simulación clínica y posteriormente fue revisado por estudiantes de noveno semestre de la carrera de enfermería, para determinar su claridad. Se realizó una prueba piloto con 5 estudiantes de noveno semestre, que no participaron como sujetos de estudio de la investigación. Presentó una confiabilidad importante con un Alfa de Crombach de 0,913 , con un puntaje promedio de acuerdo con la escala Likert de 3,92 (DS=0,56), cercano al 4, que corresponde a "de acuerdo", con un puntaje mínimo de 2,67 y un máximo de 5. Los promedios variaron significativamente $(\mathrm{p}=0,035)$ entre mujeres $(X=3,88$; $D S=0,55)$ y hombres $(X=4,19$; $\mathrm{DS}=0,48)$. Esta escala permitió medir la percepción de la importancia de la simulación clínica para desempeño del estudiante en su práctica clínica.

Para el análisis estadístico los datos fueron codificados e ingresados al programa MS Excel de Office 365, y analizados con el programa SPSS v.15. Se utilizaron medidas de tendencia central y de variabilidad para el análisis descriptivo de las variables: promedios, desviación estándar, máximo y mínimo. Para la asociación entre variables con distribución normal se empleó pruebas paramétricas como Tde Student, Anova, correlación de Pearson, y no paramétricas para las variables con distribución no normal, como Mann-Whitney, 
KruskalWallis y correlación de Spearman, sin embargo, las asociaciones realizadas no obtuvieron significancia estadística, por lo que no se presentan estos resultados.

Respecto al abordaje cualitativo se utilizó muestreo por conveniencia, los participantes fueron seleccionados, de acuerdo a los requisitos establecidos en la fase cuantitativa, además se contactaron los participantes que contaban con las características suficientes para obtener un buen relato de acuerdo a los objetivos planteados, de tal forma de tener apreciaciones acabadas de sus impresiones frente a esta metodología y por ello fueron catalogados como informantes claves.

Se realizó entrevista en profundidad a cada informante clave, alcanzando saturación de los discursos a la octava entrevista realizada, agregando dos participantes adicionales para corroborar el cumplimiento de la saturación de los datos en base a las temáticas planteadas en la entrevista para develar el fenómeno de estudio y el objetivo de esta fase de la investigación, obteniendo finalmente la participación de 10 estudiantes.

Los discursos fueron grabados y transcritos fielmente, realizando un análisis de contenido, mediante reducción fenomenológica. Para cautelar la calidad de los datos cualitativos se realizó triangulación por investigadores.

La rigurosidad científica, en el caso de la aproximación cualitativa, se garantizó utilizando criterios de rigor según Guba y Lincoln ${ }^{(16)}$ : respecto del valor de verdad, los datos fueron recolectados de acuerdo a los relatos de los participantes y analizados siguiendo reducción fenomenológica; respecto a la aplicabilidad, la metodología fenomenológica fue adecuada para obtener los relatos subjetivos de los participantes y develar el fenómeno de estudio; para garantizar la consistencia en los relatos, se exploraron todos los temas relevantes para el fenómeno de estudio, hasta la saturación de los datos; finalmente, para asegurar la neutralidad, los investigadores se mantuvieron en todo momento al margen de prejuicios y juicios referente a los datos obtenidos en la investigación.

Lo antes expuesto da fe de la rigurosidad y veracidad de los resultados del estudio y destacan que la credibilidad (triangulación por los investigadores), dependencia (réplica paso a paso, permitiendo juzgar el grado de correspondencia con otros contextos), confirmabilidad (ejercicio de reflexión y consenso con otros investigadores) y transferibilidad (recogida abundante información descriptiva y desarrollo de descripciones minuciosas), se utilizaron como referentes de validez para los estudios cualitativos.

Tanto en la aproximación cuantitativa como cualitativa, se aplicaron las consideraciones éticas, donde el desarrollo del presente estudio revistió mínimos riesgos para los estudiantes, como por ejemplo, la utilización de tiempo personal para concertar la entrevista. En concordancia con lo anterior, el estudio fue aprobado por el comité ético científico institucional ${ }^{(17)}$ y previo a la recogida de datos, los participantes firmaron un consentimiento informado.

\section{RESULTADOS}

Caracterización sociodemográfica de los/as estudiantes: La población estaba compuesta principalmente por estudiantes de sexo femenino $(85,7 \%)$, solteras $(94,3 \%)$, sin hijos $(96,2 \%)$, del sector urbano $(91,5 \%)$, vivían arrendando $(52,8 \%)$ y acompañadas $(77,4 \%)$, la familia a la que pertenecían era monoparental $(21,4 \%)$ y biparental $(54,4 \%)$. Provenían de colegio particular subvencionado $(58,5 \%)$, para postular a la universidad cursaron algún tipo de preuniversitario (74\%), como droga lícita señalaron consumo de alcohol $(71,1 \%)$ e ilícita consumo de marihuana $(2,7 \%)$, además de señalar "otros" (10,8\%), destacando el porcentaje global para la variable "Consumo de sustancias" con un $86,7 \%$, presentaban redes de apoyo compuestas por la familia materna, amigos, pareja, iglesia etc. $(92,5 \%)$ (Tabla 1).

Todas las aseveraciones del "Cuestionario evaluación de percepción sobre utilización metodología simulación clínica" presentaron mayores porcentajes de respuesta a partir de los 3 puntos (ni de acuerdo ni en desacuerdo); las aseveraciones $1 \mathrm{y}$ 8 referidas a "enfrentar la práctica clínica" y "el logro de los objetivos de aprendizaje", respectivamente, obtuvieron mayor porcentaje de respuesta $(57,5 \%)$ en el puntaje 4 (de acuerdo); la aseveración 15 referida a "la inclusión de la simulación clínica en las mallas curriculares", tuvo mayor porcentaje de respuesta en el puntaje 5 (totalmente de acuerdo) (Tablas 2). Los estadísticos descriptivos del cuestionario están señalados en la Tabla 3. 
Tabla 1. Distribución frecuencial y porcentual de participantes según variables sociodemográficas, abordaje cuantitativo, Valdivia, Chile, agosto 2015-diciembre 2016 ( $\mathrm{n}=106)$.

\begin{tabular}{|c|c|c|c|}
\hline \multicolumn{2}{|c|}{ Variables sociodemográficas } & \multirow{2}{*}{$\begin{array}{c}\text { Fr } \\
90\end{array}$} & \multirow{2}{*}{$\frac{\%}{85,71}$} \\
\hline Sexo & Femenino & & \\
\hline & Masculino & 15 & 14,29 \\
\hline & Total & $105^{*}$ & 100,00 \\
\hline \multirow[t]{3}{*}{ Sector donde proviene } & Rural & 9 & 8,49 \\
\hline & Urbano & 97 & 91,51 \\
\hline & Total & 106 & 100,00 \\
\hline \multirow[t]{5}{*}{ Usted Vive en } & Casa de familia de origen & 34 & 32,08 \\
\hline & Arrendatario & 56 & 52,83 \\
\hline & Pensión & 8 & 7,55 \\
\hline & Casa de familiares & 8 & 7,55 \\
\hline & Total & 106 & 100,00 \\
\hline \multirow[t]{3}{*}{ Usted Vive } & Solo & 24 & 22,64 \\
\hline & Acompañado & 82 & 77,36 \\
\hline & Total & 106 & 100,00 \\
\hline \multirow[t]{4}{*}{ Estado Civil } & Soltero & 100 & 94,34 \\
\hline & Casado & 1 & 0,94 \\
\hline & Conviviente & 5 & 4,72 \\
\hline & Total & 106 & 100 \\
\hline \multirow[t]{3}{*}{ Presencia hijos } & No & 102 & 96,23 \\
\hline & $\mathrm{Si}$ & 4 & 3,77 \\
\hline & Total & 106 & 100 \\
\hline \multirow[t]{4}{*}{ Enseńanza media } & Colegio Particular & 10 & 9,43 \\
\hline & Colegio Subvencionado & 62 & 58,49 \\
\hline & Colegio público & 34 & 32,08 \\
\hline & Total & 106 & 100 \\
\hline \multirow[t]{4}{*}{ Redes Apoyo } & Familia materna & 4 & 3,77 \\
\hline & Amigos Personales & 4 & 3,77 \\
\hline & Otros (familia materna, pareja, iglesia. etc.) & 98 & 92,45 \\
\hline & Total & 106 & 100 \\
\hline \multirow[t]{7}{*}{ Tipo de familia } & Simple & 3 & 2,9 \\
\hline & Monoparental & 22 & 21,4 \\
\hline & Biparental & 56 & 54,4 \\
\hline & Nuclear & 13 & 12,6 \\
\hline & Extensa & 5 & 4,9 \\
\hline & Compuesta & 4 & 3,9 \\
\hline & Total & $103^{*}$ & 100 \\
\hline \multirow[t]{3}{*}{ Preuniversitario } & $\mathrm{Si}$ & 77 & 74 \\
\hline & No & 27 & 26 \\
\hline & Total & $104^{*}$ & 100 \\
\hline \multirow[t]{5}{*}{ Consumo de sustancias } & No consume & 11 & 13,3 \\
\hline & Alcohol & 59 & 71,1 \\
\hline & Mariguana & 4 & 4,8 \\
\hline & Otros & 9 & 10,8 \\
\hline & Total & $83^{*}$ & 100 \\
\hline
\end{tabular}

* Existen respuestas no consignadas por los participantes 
Tabla 2. Distribución de la población abordaje cuantitativo según respuesta del Cuestionario evaluación de percepción sobre utilización metodología simulación clínica, Valdivia, Chile, agosto 2015-diciembre 2016 ( $\mathrm{n}=106)$.

\begin{tabular}{|c|c|c|c|c|c|c|c|c|c|c|c|c|}
\hline \multirow[t]{2}{*}{ Items } & \multicolumn{2}{|c|}{$\begin{array}{l}\text { Totalmente en } \\
\text { desacuerdo } \\
\text { (1 pto.) }\end{array}$} & \multicolumn{2}{|c|}{$\begin{array}{l}\text { En desacuerdo } \\
\text { (2 ptos) }\end{array}$} & \multicolumn{2}{|c|}{$\begin{array}{c}\text { Ni de acuerdo } \\
\text { ni en des- } \\
\text { acuerdo } \\
(3 \text { ptos })\end{array}$} & \multicolumn{2}{|c|}{$\begin{array}{l}\text { De acuerdo } \\
\text { (4 ptos) }\end{array}$} & \multicolumn{2}{|c|}{$\begin{array}{l}\text { Totalmente de } \\
\text { acuerdo } \\
\text { (5 ptos) }\end{array}$} & \multicolumn{2}{|c|}{ Total } \\
\hline & $\mathbf{N}^{\circ}$ & $\%$ & $\mathbf{N}^{\circ}$ & $\%$ & $\mathbf{N}^{\circ}$ & $\%$ & $\mathbf{N}^{\circ}$ & $\%$ & $\mathbf{N}^{\circ}$ & $\%$ & $\mathbf{N}^{\circ}$ & \%* \\
\hline 1 & 0 & 0,0 & 7 & 6,6 & 24 & 22,6 & 61 & 57,5 & 14 & 13,2 & 106 & 100 \\
\hline 2 & 0 & 0,0 & 2 & 1,9 & 10 & 9,5 & 60 & 57,1 & 33 & 31,4 & 105 & 100 \\
\hline 3 & 1 & 1,0 & 8 & 7,6 & 32 & 30,5 & 39 & 37,1 & 25 & 23,8 & 105 & 100 \\
\hline 4 & 0 & 0,0 & 2 & 1,9 & 12 & 11,4 & 64 & 61,0 & 27 & 25,7 & 105 & 100 \\
\hline 5 & 2 & 1,9 & 8 & 9,4 & 40 & 37,7 & 39 & 36,8 & 17 & 16,0 & 104 & 100 \\
\hline 6 & 0 & 0,0 & 0 & 0,0 & 5 & 4,80 & 50 & 47,6 & 50 & 47,6 & 105 & 100 \\
\hline 7 & 3 & 2,8 & 8 & 7,5 & 35 & 33,0 & 46 & 43,4 & 14 & 13,2 & 106 & 100 \\
\hline 8 & 1 & 0,9 & 4 & 3,8 & 24 & 22,6 & 61 & 57,5 & 16 & 15,1 & 106 & 100 \\
\hline 9 & 1 & 1,0 & 2 & 1,9 & 24 & 22,9 & 49 & 46,7 & 29 & 27,6 & 105 & 100 \\
\hline 10 & 0 & 0,0 & 5 & 4,7 & 45 & 42,5 & 37 & 34,9 & 19 & 17,9 & 106 & 100 \\
\hline 11 & 0 & 0,0 & 5 & 4,7 & 24 & 22,6 & 53 & 50,0 & 24 & 22,6 & 106 & 100 \\
\hline 12 & 0 & 0,0 & 3 & 2,8 & 22 & 20,8 & 44 & 41,5 & 37 & 34,9 & 106 & 100 \\
\hline 13 & 2 & 1,9 & 17 & 16,0 & 34 & 32,1 & 37 & 34,9 & 16 & 15,1 & 106 & 100 \\
\hline 14 & 1 & 0,9 & 7 & 6,7 & 14 & 13,3 & 49 & 46,7 & 34 & 32,4 & 105 & 100 \\
\hline 15 & 0 & 0,0 & 1 & 1,0 & 8 & 7,6 & 41 & 39,0 & 55 & 52,4 & 105 & 100 \\
\hline
\end{tabular}

* $100 \%$ de los que contestaron el ítem

Tabla 3. Puntajes Cuestionario evaluación de percepción sobre utilización metodología simulación clínica. Valdivia, Chile, agosto 2015-diciembre 2016.

\begin{tabular}{ccccc}
\hline $\mathbf{N}$ & Mínimo & Máximo & Media & DS \\
\hline 106 & 2,67 & 5,00 & 3,9157 &, 55716 \\
\hline
\end{tabular}

La distribución de algunas aseveraciones con la suma de los porcentajes de respuesta alcanzados en las respuestas "de acuerdo" y "totalmente de acuerdo" se detalla continuación: 2) "La simulación me ha ayudado a integrar teoría y práctica” (88,5\%); 4) "me sentí comprometido en mi autoaprendizaje" $(86,7 \%)$; 6) "la metodología utilizada ayuda a utilizar el razonamiento crítico y la toma de decisiones" (95,2\%); 9) "las experiencias vividas con la aplicación de la metodología fueron importantes en mi aprendizaje" $(74,3 \%) ; 12)$ "la utilización de la metodología ha contribuido a reconocer la responsabilidad social que me compete" (76,4\%); 14) "en general la experiencia de trabajar con la simulación clínica ha sido satisfactoria” (79,1\%); 15) "la utilización de esta metodología debería incluirse en las mallas curriculares de las carreras del área de la salud en forma permanente" $(91,4 \%)$.

Las aseveraciones con menos porcentajes de respuestas, en la suma de la clasificación "en desacuerdo" y "ni de acuerdo ni en desacuerdo" fueron: 1) después de empezar a utilizar esta metodología sentí que mi forma de enfrentar las situaciones en la práctica clínica había evolucionado positivamente" (29,2\%) y 10) "la interacción con la simulación ha mejorado mi competencia clínica, con relación al grado de desarrollo de habilidades y destrezas adquiridas" (47,2\%) (Tabla 2). 
Desde el punto de vista cualitativo se develaron 3 categorías y 12 subcategorías (Tabla 4):

Categoría "Sentimientos y emociones vivenciados": Los estudiantes expresaron que los principales sentimientos y emociones emanaban de la experiencia vivida, reforzando la responsabilidad de su actuar profesional, al disminuir el temor a equivocarse y producir daño, con sensación de seguridad, confianza y satisfacción en las acciones con el equipo, familia y paciente:

"A uno como estudiante, le permiten aprender..., o sea, saber cómo enfrentar situaciones con una postura más...no tan nerviosa, no tan asustados, sino con una postura más..., con ojo más crítico" (E5).

"Dentro del campo clinico como que uno tal vez se sentía como más seguro y confiado de que hay cosas que uno ya ha experimentado en cierto modo y lo otro de no tener miedo" (E8).

"Como que uno se siente como más orgullosa, más satisfecha de poder llevar a la práctica lo que vio en la práctica simulada" (E3).

Categoría "Importancia y valor que le otorga": Los informantes develaron el valor de contar con este tipo de entrenamiento, planteando que debe ser reconocido como sello de la formación, indicando la relevancia de la retroalimentación proporcionada por el docente en el debriefing, aportando desde su experiencia y la de los compañeros al compartir las perspectivas individuales, resaltando su importancia y beneficio como método para favorecer el éxito en la resolución de las situaciones planteadas.

La posibilidad de atreverse a interactuar en la resolución de problemas planteados en escenarios de simulación fue indicada como importante, otorgándoles la oportunidad de equivocarse al enfrentar situaciones que habitualmente no están a su alcance y poder practicar, sin provocar daños a terceros ni lesionar su aspecto emocional.

Participar en actividades de simulación les permite adquirir herramientas de afrontamiento, extendiéndose este beneficio a la vida personal, donde la memoria generada con los aprendizajes, les sirve para afrontar situaciones:

"Yo creo que sería bueno que esto permanezca en el tiempo, que se diga en enfermería de la UACh se enseña a través de simulación, yo creo que sería parte de la identidad, que debería formar parte de la identidad del estudiante de enfermería de la Austral" (E1).

"Da buenas herramientas... para afrontar el quehacer del campo clinico... tanto en la atención directa al paciente como en nuestra vinculación con el equipo y nos otorga herramientas para afiatar lazos"(E2).

"Cuando uno visualiza lo que hizo, el rebobinar darse el tiempo de pensar que hubiese hecho de otra manera, como hubiese actuado mejor, o que hice bien, y que hice mal, que tengo que mejorar o reforzar, esa es la mejor parte de todo" (E4).

Tabla 4. Categorías y subcategorías aproximación cualitativa, Valdivia, Chile, agosto 2015-diciembre 2016.

\begin{tabular}{ll}
\hline Categorías & Subcategorías \\
\hline Sentimientos y emociones vivenciados & Satisfacción \\
& Seguridad \\
& Confianza \\
& Orgullo/compromiso \\
\hline Importancia y valor que le otorga & Identidad \\
& Retroalimentación \\
& Oportunidad de equivocarse \\
& Oportunidad de practicar \\
& Herramientas de afrontamiento \\
\hline Ámbito de mejora al que apunta & Desarrollo de competencias \\
& Interacción con los equipos \\
& Experiencia \\
\hline
\end{tabular}


Categoría "Ámbito de mejora al que apunta": Los participantes revelaron que la utilización de simulación clínica les confirió algún grado de experiencia para la resolución de situaciones difíciles, favoreciendo el auto conocimiento, pudiendo reconocer cómo se desenvuelven frente a dichos escenarios, identificando sus fortalezas y brechas en las que deben trabajar, explorando, también, la interacción con los equipos de trabajo y poder extrapolar estas habilidades a la vida personal: "Con las simulaciones uno va destacando la importancia que tienen también las relaciones humanas, las relaciones en el equipo, entonces uno le entrega importancia también a esos aspectos, no solamente a lo asistencial" (E3).

"Hay cosas que las vemos en la teoría pero que nunca las vemos en la práctica realmente y que...llegado el momento...ya tenemos la experiencia de haberlas vivido por esta metodología" (E6).

"Uno puede poner en práctica competencias necesarias frente a situaciones adversas, a situaciones de la vida diaria que se presenten, permite también actuar a tiempo, rápido, con seguridad y también manejar las relaciones humanas" (E8).

\section{DISCUSIÓN}

Las características sociodemográficas se comportaron de acuerdo con la distribución que han tenido históricamente las carreras de enfermería, predominando el sexo femenino y de procedencia de zonas urbanas, diferenciándose de otros hallazgos en que la mayor parte de ellos no pertenecen al mismo lugar donde se encuentra la casa de estudios ${ }^{(18)}$.

Por otro lado, pertenecen al sector urbano, la mayoría no vive con su familia de origen, presentan alta frecuencia de consumo de alcohol, acompañado de algún grado de consumo de marihuana y otros tipos de sustancias; pero a pesar de ello reconocen contar con redes de apoyo y en un alto porcentaje viven acompañados. La presencia de consumo se ve reflejado en diferentes estudios como en México donde muestran que las principales sustancias consumidas son el alcohol y tabaco en porcentaje importante ${ }^{(19,20)}$. Es necesario destacar la relevancia sobre el consumo de alcohol y marihuana, además de otro tipo de sustancias, pues, como factor de riesgo, podría repercutir negativamente en el rendimiento académico, además de experimentar las conocidas consecuencias lesivas en la salud de los estudiantes, por ello debe ser abordado por la institución, a fin de mitigar dichos efectos y favorecer el bienestar de los jóvenes para un buen desempeño académico.

Hay aspectos del análisis tanto de los hallazgos cuantitativos como cualitativos que concuerdan. La gran mayoría de los estudiantes estaban de acuerdo con la metodología de simulación clínica, valorando la adecuada formulación y desarrollo de los escenarios de simulación; reconocieron que les ayudaba a mejorar la autonomía en el aprendizaje para integrar los conocimientos a la práctica diaria, destacando que la metodología potencia notablemente habilidades y destrezas clínicas, desarrollo del pensamiento crítico y toma de decisiones. Esto está confirmado por diversos estudios que hablan del desarrollo de habilidades, autoconfianza y satisfacción en la metodología ${ }^{(21-24)}$, aspectos que favorecen el desarrollo personal, el desempeño profesional y el juicio clínico y que deben ser potenciados en la educación superior $y$, en específico, en las carreras de las ciencias de la salud.

Además, se observa que la metodología de simulación clínica brinda el incentivo necesario para lograr que los estudiantes se involucren en la experiencia, yendo más allá de los ámbitos teóricos o prácticos, visualizando aspectos de responsabilidad social como profesional de la salud en formación. Por lo tanto, trabajar con la metodología de simulación ha sido satisfactorio para los estudiantes, manifestando estar de acuerdo con su inclusión de manera permanente en las mallas curriculares de las carreras del área de la salud, como lo demuestra un estudio realizado en estudiantes de Farmacia, que preferían que la simulación clínica se incorporara tempranamente en el currículo, incluso antes que otras metodologías de enseñanza aprendizaje $\mathrm{e}^{(25)}$.

Destaca la percepción de aumento del nivel de seguridad y confianza de los estudiantes sobre su desempeño al enfrentar situaciones complejas de la atención en contextos reales, además de contar con la posibilidad de equivocarse en un entorno simulado, aprendiendo del error y entrenar competencias como liderazgo, trabajo en equipo, manejo de conflictos, comunicación efectiva, distribución de recursos, toma de decisiones, etc., lo que se ha descrito en algunos estudios, no solo en estudiantes de enfermería, sino que también en otras carreras del área de la salud ${ }^{(25,26)}$. 
Desde el punto de vista fenomenológico los estudiantes manifiestan emociones positivas, atribuidas a las experiencias de simulación clínica que influían en su disposición a enfrentar, no solo actividades clínicas, sino también situaciones de vida personal. En este proceso, surgen sentimientos de satisfacción frente a la metodología, porque al saber qué errores se han cometido y cómo pueden ser subsanados, se sienten más preparados para enfrentar una situación real. Estas experiencias, que las pueden extender a su vida diaria, se transforman y reconocen como un aprendizaje significativo. Estos hallazgos se reafirman con los encontrados en algunos estudios donde, además, se señala que los estudiantes muestran sentimientos de orgullo al aplicar lo aprendido, acompañado por cierta ansiedad y preocupación por mejorar ${ }^{(27)}$.

Según los informantes, el periodo reflexivo desarrollado en el debriefing es un momento fundamental para internalizar las competencias alcanzadas y poder analizar la mejor manera para enfrentar la situación planteada en la actividad de simulación, señalando que la guía del docente les resulta clave en el proceso. Varios estudios concuerdan con que esta etapa de la simulación clínica se traduce en resultados muy positivos para los estudiantes ${ }^{(28,29)}$, apoyado en el logro de aprendizaje reflexivo que permite reconocer aspectos positivos y áreas de mejora para el desempeño en futuras intervenciones.

Los estudiantes le otorgan gran valor a la oportunidad de practicar, poder equivocarse sin dañar a los pacientes y con ello desarrollar habilidades y aprender a afrontar situaciones. Destacan que la adquisición de experiencia y el autoconocimiento son relevantes para su desempeño posterior, sobre todo en aspectos que interviene el factor humano, donde el saber ser es clave para un buen resultado. Esto se observa como un aspecto positivo para la salud mental de las personas, pues influiría directamente en la autoestima y autoconcepto. Esto está claramente descrito en un estudio realizado en Corea del Sur, donde además resultaba positivo para la eficacia colectiva y se relacionaba negativamente al estrés percibido ${ }^{(30)}$.

Según los informantes la simulación clínica se muestra como una metodología eficiente, que optimiza el proceso de enseñanza aprendizaje, favoreciendo además el desarrollo de habilidades no procedimentales relacionadas con competencias genéricas, hechos que concuerdan con diversos estudios en los que se ha demostrado ampliamente su beneficio y efectividad ${ }^{(31-34)}$.

Por otra parte, implica experimentar sentimientos que generan memoria emocional que permiten forjar recuerdos permanentes, favoreciendo el aprendizaje significativo. Además de observase como posible factor protector frente a ciertas alteraciones emocionales provocadas por el desarrollo de actividades clínicas, se visualiza como aspecto favorecedor para la mantención de una buena salud mental en el ámbito académico, aspecto que podría ser profundizado en futuros estudios.

Sería importante la revisión de los currículos de las carreras para poder insertar esta metodología tempranamente permitiendo que el estudiante visualice su desarrollo académico, profesional y personal, además de potenciar durante el transcurso de la carrera aquellos aspectos positivos que esta metodología representa para los estudiantes.

Como limitaciones del estudio es posible señalar que si bien, en el caso de la aproximación cuantitativa, se contó con un universo de 106 estudiantes, este pudiera ser insuficiente para realizar análisis estadístico con alta sensibilidad, por lo tanto, se seleccionaron los estadígrafos más apropiados para el análisis los datos. Además, otra limitación podría estar relacionada con el hecho de no haber podido incluir en el estudio a estudiantes de otras carreras del área de la salud, lo que podría haber enriquecido la obtención de resultados y la posterior discusión y conclusiones de este estudio.

Es necesario señalar que incluir solo a los estudiantes de la carrera de enfermería podría haber influido, de cierta manera, en las respuestas de los estudiantes, transformándose en un posible sesgo al ser los investigadores, además, docentes de la misma disciplina. A fin de evitar lo anterior, en la fase de reclutamiento no se consideró una muestra y se incluyó el universo de estudiantes que habían cursado $7^{\circ}$ y $8^{\circ}$ semestre en cada cohorte. Además se optó por recolectar los datos en modalidad de cuestionarios auto aplicados y en el caso de las entrevistas, estas fueron realizadas por el investigador que no tuvo actividades directas con aquellos estudiantes durante el periodo de recolección de datos. 


\section{CONCLUSIONES}

Los estudiantes destacan la importancia de la metodología de simulación clínica como preparación previa al momento de enfrentarse a situaciones difíciles o desconocidas en la atención y la transcendencia para la satisfacción usuaria, la seguridad del paciente $y$, por consiguiente, la propia. Le confieren un alto valor para su desarrollo profesional y personal, y al logro de un aprendizaje significativo, que puede ser extrapolado al campo clínico, su utilización genera diversos sentimientos, reconociéndola como parte de la identidad del estudiante de Enfermería de la Universidad Austral de Chile.

La metodología resulta satisfactoria para los estudiantes, generando memoria emocional, convirtiéndose en una herramienta fundamental en la formación profesional para el desarrollo, incremento y optimización de habilidades procedimentales y no procedimentales, como el pensamiento crítico y juicio clínico, que apuntan al logro de competencias primordiales para el ejercicio profesional, desde una perspectiva ética, beneficiando directamente a estudiantes, pacientes y equipo de trabajo en escenarios reales y fortaleciendo con ello la calidad y seguridad de la atención brindada.

Conflictos de interés: Los autores declaran no tener ningún conflicto de interés.

\section{REFERENCIAS}

1. Guinez-Molinos S, Maragaño P, Gomar-Sancho C. Simulación clínica colaborativa para el desarrollo de competencias de trabajo en equipo en estudiantes de medicina. Rev méd Chile [Internet]. 2018 [citado 15 jun 2018]; 146(5): 643-652. Disponible en: http://dx.doi.org/10.4067/s003498872018000500643.

2. Ronquillo TL, Cabrera GC, Barberán CJ. Competencias profesionales: desafíos en el proceso de formación profesional. Opuntia Brava [Internet]. 2019 [citado 3 dic 2021]; 11(Especial 1): 1-2. Disponible en: http://200.14.53.83/index.php/ opuntiabrava/article/view/653

3. López-Entrambasaguas OM, Martínez-Yebenes R, Calero-García MJ, Granero-Molina J, MartínezLinares JM. Newly Qualified Nurses' Perception of Their Competency Achievement on Leaving University: A Qualitative Study. Int J Environ
Res Public Health [Internet]. 2019 [citado 21 dic 2019]; 16(21): 4284. Disponible en: http://dx.doi. org/10.3390/ijerph16214284.

4. Ayala-Guzmán CI, Verde-Flota E, Monroy-Rojas A, Contreras-Garfias ME, Rivas-Espinosa JG. Proyecto Tuning: competencias genéricas y exigencias laborales en egresados de una licenciatura de enfermería en México. Rev Enferm IMSS [Internet]. 2017 [citado 3 dic 2021]; 25(1): 3746. Disponible en: https://pesquisa.bvsalud.org/ portal/resource/pt/biblio-1031313

5. Rourke S. How does virtual reality simulation compare to simulated practice in the acquisition of clinical psychomotor skills for pre-registration student nurses? A systematic review. Int J Nurs Stud [Internet]. 2019 [citado 15 dic 2019]; 102: 103466. Disponible en: http://dx.doi.org/10.1016/ j.ijnurstu.2019.103466.

6. Villca S. Simulación clínica y seguridad de los pacientes en la educación médica. Rev Cien Tec In [Internet]. 2018 [citado 25 ene 2019]; 16(18): 7588. Disponible en: http://bit.ly/2PzMCGM

7. Urra E, Sandoval S, Irribarren F. El desafío y futuro de la simulación como estrategia de enseñanza en enfermería. Inv Ed Méd [Internet]. 2017 [citado 25 jun 2018]; 6 (22): 119-125. Disponible en: http://dx.doi.org/10.1016/j.riem.2017.01.147.

8. Rodríguez CM. La educación médica en tiempos del covid-19. Rev méd hered [Internet]. 2020 [citado 5 dic 2021]; 31(3): 143-7. Disponible en: https://revistas.upch.edu.pe/index.php/RMH/ article/view/3802

9. Kohn L, Corrigan J, Donaldson M. To err is human: building a safer health system. Comite on Health Care in America. Institute of Medicine. Washington (DC): National Academy Press; 1999. Disponible en: http//dx.doi.org/10.17226/9728.

10. Escudero E, Avendaño B-AM, Domínguez CK. Simulación clínica y seguridad del paciente: integración en el currículo de enfermería. Sci Med [Internet]. 2018 [citado 4 dic 2021]; 28(1): ID28853. Disponible en: http://dx.doi.org/10.15448/1980 $-6108.2018 .1 .28853$

11. Código Sanitario. Ley $\mathrm{N}^{\circ}$ 19.536. Ministerio de salud subsecretaría de salud [Internet]. Diario Oficial de Chile (16-12- 1997). Disponible en: https://bit.ly/3kTlrDK

12. Moya P, Ruz M, Parraguez E, Carreño V, Rodríguez A, Froes P. Efectividad de la simulación en la educación médica desde la perspectiva de seguridad de pacientes. Rev méd Chile [Internet]. 2017 [citado 15 ago 2018]; 145(4): 514-526. Disponible en: http://dx.doi.org/10.4067/S003498872017000400012.

13. de Dios M, Varela I, Braschi L, Sánchez E. Estrés 
en estudiantes de enfermería. Educ Med Super [Internet]. 2017 [citado 30 ago 2018]; 31(3): 110123. Disponible en: http://dx.doi.org/10.1016/ S1130-8621(05)71136-0.

14. Verissimo R, Ferreira W. O Método fenomenológico nas pesquisas em saúde no Brasil: Uma análise de produção científica. Trab Educ Saúde [Internet]. 2018 [citado 20 ene 2019]; 16(3): 1421-1441. Disponible en: http://de.doi.org/10.1590/19817746-sol00162.

15. Juarez-Hernandez LG, Tobón S. Análisis de los elementos implícitos en la validación de contenido de un instrumento de investigación. Rev Espac [Internet]. 2018 [citado 5 dic 2021]; 39(53). Disponible en: https://www.revistaespacios.com/ cited2017/cited2017-23.pdf

16. Guba E, Lincoln Y. Naturalistic Inquiry [Internet]. London, Sage. 1985: 37-40. Disponible en: https:// bit.ly/39NotmH

17. Fredes M, Vergara P, Etcheverry J. Actualización de las pautas CIOMS. ARS med [Internet]. 2017 [citado 4 dic 2021]; 42(3): 55-59. Disponible en: http://dx.doi.org/10.11565/arsmed.v42i3.742

18. Astudillo A, López MA, Cádiz V, Fierro J, Figueroa A, Vilches N. Validación de la encuesta de calidad y satisfacción de simulación clínica en estudiantes de enfermería. Cienc enferm [Internet]. 2017 [citado 30 oct 2019]; 23(2): 133-145. Disponible en: http:// dx.doi.org/10.4067/S0717-95532017000200133

19. Medina J, Ortiz L. Prevalencia y conocimientos de estudiantes de enfermería de una universidad pública sobre el cuidado a la persona que consume drogas lícitas. Revenf [Internet]. 2017 [citado 5 nov 2019]; (32): 1-13. Disponible en: http:// dx.doi.org/10.15517/revenf.v0i32.24399

20. González P, Hernández EK, Rodríguez LA, Castillo R, Salazar J, Camacho JU. Percepción de riesgo ante el consumo de alcohol y tabaco en estudiantes de ciencias de la salud de Saltillo. Enf Global [Internet]. 2019 [citado 4 dic 2021]; 18(4): 398422. Disponible en: https://revistas.um.es/eglobal/ article/view/351381

21. Lubbers J, Rossman C. Satisfaction and selfconfidence with nursing clinical simulation: Novice learners, medium-fidelity, and community settings. Nurse Educ Today [Internet]. 2017 [citado 21 nov 2019]; 48: 140-144. Disponible en: http://dx.doi. org/10.1016/j.nedt.

22. Cabrera TAA, Kempfer SS. Simulación Clínica en la enseńanza de la Enfermería: experiencia de estudiantes en Chile. Texto contexto enferm [Internet]. 2020 [citado 6 dic 2021]; 29(Spe): e20190295. Disponible en: https://doi.org/10.1590/ 1980-265X-TCE-2019-0295

23. Hustad J, Johannesen B, Fossum M, Hovland OJ.
Nursing students' transfer of learning outcomes from simulation-based training to clinical practice: a focus-group study. BMC Nurs [Internet]. 2019 [citado 25 nov 2019]; 18:53. Disponible en: https://doi.org/10.1186/s12912-019-0376-5

24. Reevs NE, Waite MC, Tuttle N, Bialocerkowski A. Simulated patient contributions to enhancing exercise physiology student clinical assessment. Skills Adv Simul [Internet]. 2019 [citado 26 dic 2019]; 4(1): 15. Disponible en: http://dx.doi. org/10.1186/s41077-019-0097-6.

25. Dahri K, MacNeil K, Chan F, Lamoureux E, Bakker $\mathrm{M}$, Seto K, et al. Curriculum integration of virtual patients. Curr Pharm Teach Learn [Internet]. 2019 [citado 15 dic 2019]; 11(12): 1309-1315. Disponible en: http://dx.doi.org/10.1016/j.cptl. 2019.09.007.

26. Duprey MD, Dunker KS. Megacode Simulation Training in Undergraduate Nursing Education. Nurs Educ Perspect [Internet]. 2019 [citado 13 dic 2019]; 1-2. Disponible en: http://dx.doi. org/10.1097/01.NEP.0000000000000589.

27. Behrens CC, Dolmans DH, Gormley GJ, Driessen EW. Exploring undergraduate students achievement emotions during ward round simulation: a mixed-method study. BMC Med Educ [Internet]. 2019 [citado 30 nov 2019]; 19(1): 316. Disponible en: https://doi.org/10.1186/s12909019-1753-1

28. Harde N, Lemoine J, Harwood R. Psychological outcomes of debriefing healthcare providers who experience expected and unexpected patient death in clinical or simulation experiences: A scoping review. J Clin Nurs [Internet]. 2020 [citado 13 ene 2020]; 29(3-4): 330-346. Disponible en: https:// doi.org/10.1111/jocn.15085

29. Mano L, Mazzo A, Torres JR, Filho C, Goncalves VP, Ueyama J, et al. The Relation of Satisfaction, Self-Confidence and Emotion in a Simulated Environment. Int J Nurs Educ Scholarsh [Internet]. 2019 [citado 27 dic 2019]; 16(1). Disponible en: https://doi.org/10.1515/ijnes-2018-0009.

30. Kim MY, Park S. Associations of stress, self-esteem, and collective efficacy with flow in simulation among nursing students: A descriptive crosssectional study. Nurse Educ Today [Internet]. 2018 [citado 18 dic 2019]; 71: 193-197. Disponible en: https://doi.org/10.1016/j.nedt.2018.09.033

31. Rueda D, Arcos ME, Alemán ME. Simulación clínica, una herramienta eficaz para el aprendizaje en ciencias de la salud. Rev Publicando [Internet]. 2017 [citado 5 dic 2021]; 13(2): 225-43. Disponible en: https://revistapublicando.org/revista/index. $\mathrm{php} / \mathrm{crv} /$ article/view/838

32. Ayala-Valladolid D, Espinoza-Moreno T. Utilidad 
de la simulación clínica para lograr competencias en estudiantes de enfermería en tiempos de COVID-19. Rev cuba Enferm [Internet]. 2020 [citado 5 dic 2021]; 36. Disponible en: http:// www.revenfermeria.sld.cu/index.php/enf/article/ view/3946

33. Fuentes C, Corvetto M, Altermatt F, Delfino A. Incorporación de la simulación en el entrenamiento del manejo de recursos en crisis, experiencia del programa de anestesiología de la Pontificia
Universidad Católica de Chile. ARS MEDICA [Internet]. 2017 [citado 30 dic 2019]; 42(1): 2733. Disponible en: http://dx.doi.org/10.11565/ arsmed.v42i1.393

34. Pérez M, Areco J, Noya B, Rodríguez AM. Listas de chequeo: crisis en sala de operaciones. Anest Analg Reanim [Internet]. 2017 [citado 4 ene 2020]; 30(2): 2-12. Disponible en: https://bit. ly/3ouKfUD 